\title{
Factors associated with delivering premature and/or low birth weight infants among pregnant HIV-positive women on antiretroviral treatment at Dr George Mukhari Hospital, South Africa
}

\author{
NN Gibango ${ }^{a}$, S Mda ${ }^{a}$ and TS Ntulib* \\ a Department of Paediatrics and Child Health, Sefako Makgatho Health Sciences University, Pretoria, South Africa \\ ${ }^{b}$ Department of Public Health, University of Limpopo (Turfloop Campus), Sovenga, South Africa \\ *Corresponding author, email: tsntuli@hotmail.com
}

Background: Prematurity and low birthweight (LBW) deliveries amongst pregnant women infected with the human immunodeficiency virus (HIV) remain a challenge worldwide. The association between prematurity, LBW and antiretroviral therapy (ART) or prophylactic antiretroviral drug (ARV) exposure in pregnancy is unclear. This study evaluates the risk of delivering a premature and/or LBW infant among HIV-positive pregnant women on ART or prophylactic ARV.

Methods: A cross-sectional study was conducted (April to October 2012). HIV-positive women on prophylactic ARV (dual therapy) or lifelong ART (triple therapy or HAART) were enrolled in the study. Women who did not have a documented HIV result during pregnancy, those tested before delivery and those found to be HIV-positive were considered as not exposed to ARV drugs during pregnancy. This group received a standard dose of nevirapine during labour. The control group was made up of HIV-negative women.

Results: Of the 496 mothers enrolled in the study, 59\% (288/496) were HIV-positive, of whom 72\% (206/288) were on ART or prophylactic ARV. The mean age was $27.6 \pm 6.5$ years ( 15 to 47 years). The mean gestational age (GA) was $35.9 \pm 3.6$ weeks (2442 weeks). Infants' birthweights ranged from 550 to $4900 \mathrm{~g}(2.5 \pm 0.9 \mathrm{~kg})$. HIV-positive mothers not on ART or ARV prophylaxis were likely to deliver an infant at GA $<28$ weeks $(p<0.05)$ or birthweight $<1000 \mathrm{~g}(p<0.05)$ compared with their counterparts. Conclusion: HIV-positive pregnant women not on ART or ARV prophylaxis were at a risk of delivering babies at GA $<28$ weeks or birthweight $<1000 \mathrm{~g}$. There is a need to encourage early and regular attendance for antenatal care so that HIV-positive pregnant women can be identified and have access to treatment during pregnancy.

Keywords: antiretroviral therapy exposure, HIV, pregnant women

\section{Introduction}

An estimated 36.7 million people were living with the human immunodeficiency virus (HIV) worldwide in 2015, of which most (68\%) lived in sub-Saharan Africa. ${ }^{1}$ In South Africa (SA), the national surveillance of HIV and syphilis infection found an HIV prevalence of $29.7 \%$ amongst pregnant women in $2013 .{ }^{2}$ The use of antiretroviral therapy (ART) and antiretroviral (ARV) prophylaxis for the reduction of mother-to-child transmission of HIV has been a global health strategy since 2000; however, studies have provided inconsistent findings regarding the association between premature births, low birthweight (LBW) and ARV drug exposure.

There is clear evidence of the benefits of ARV drug regimens given to pregnant women for both the mother and infant;; ${ }^{3-6}$ however, studies in developed countries have found an increased rate of advanced maternal age, LBW and premature birth in HIVpositive women on ART when compared with HIV-negative women. ${ }^{7,8} \mathrm{~A}$ meta-analysis of 10 studies revealed that the use of protease inhibitor (PI)-based ART drugs during pregnancy significantly increases risk of preterm birth. ${ }^{9}$ In contrast, a study conducted in SA found that ART drug exposure reduces very LBW and premature delivery rates. ${ }^{10}$ However, Xiao and coauthors, in their study, reported that ART drug usage did not have a significant impact on the weight of the infant. ${ }^{11}$

Given the discrepant findings, there is little or no information on the risk of LBW and premature birth in HIV-positive pregnant women on ART or ARV prophylaxis at Dr George Mukhari
Hospital. Therefore, this study aimed to evaluate the risk of delivering a premature or LBW infant amongst HIV-positive pregnant women on ART and ARV prophylaxis in our setting.

\section{Methodology}

\section{Study design}

An observational cross-sectional study was conducted at $\mathrm{Dr}$ George Mukhari Hospital (DGMH) for a period of seven months from April to October 2012.

\section{Study settings and population}

The hospital is a tertiary academic hospital of the Sefako Makgatho Health Science University previously known as the University of Limpopo (Medunsa Campus). It is located in the north of Pretoria, the capital city of South Africa. Pregnant women with complications are referred to the DGMC from surrounding primary healthcare clinics and a secondary hospital in the area. The hospital services a large community of mostly black African individuals. The data for the study were collected from the postnatal and neonatal wards of this hospital. Mothers with non-complicated vaginal and Caesarean section (CS) deliveries are sent to the postnatal ward with their babies if they are term or preterm with a weight of more than $1800 \mathrm{~g}$. Preterm babies less than $1800 \mathrm{~g}$ and sick babies are admitted to the neonatal ward. The study included all mother and baby pairs from both the postnatal ward and the neonatal ward. 


\section{Sampling technique and sample size}

A minimum sample size of 322 was calculated based on the $95 \%$ confidence level, $5 \%$ sampling error and prevalence of HIV amongst pregnant women of $30 \% .^{2}$ A consecutive sample of pregnant women who consented to participate in the study was selected every day (Monday to Friday).

\section{Exclusion criteria}

Pregnant women with a chronic medical condition, such as cardiac disease, epilepsy, renal disease, liver failure, psychiatric condition and diabetes mellitus on treatment before pregnancy, were excluded. We also excluded multiple pregnancies because of the high risk of a premature birth. Infants weighing less than $500 \mathrm{~g}$ as per WHO definition of viability or less than 24 weeks gestational age (GA) were also excluded.

\section{Data-collection methods}

Data were collected from mothers' files in the postnatal ward, the antenatal card and from a questionnaire answered verbally by the mothers. The following information was obtained: age, HIV status, CD4 cell count and mode of delivery. The infant's weight was measured with a mechanical infant scale model RGZ-20 and the gestational age was calculated using the Ballard score. Prematurity was defined as delivery before 37 completed weeks by vaginal delivery or Caesarean section.

A kit for a rapid HIV Elisa test (HIV test first response HIV card test 1-2, Kachigan, Daman, India and Pareekshack HIV Triline card test Bangalore, Karataka, India) was used by nursing staff to test the HIV status of mothers who did test for HIV during pregnancy. The HIV-positive women were then divided into two groups, those who were HIV-positive on ART (triple therapy during pregnancy) or ARV prophylaxis and those who were HIV-positive but not on ART or ARV prophylaxis. The HIV-positive not on ART group was made up of women who tested HIV-positive in the labour ward and received only a single dose of nevirapine (sdNVP) during labour, three-hourly zidovudine (AZT) until delivery and then tenofovir (TDF)/emtracitabine (FTC) postdelivery. Those mothers on dual therapy were on daily AZT during pregnancy and received the sdNVP during labour plus three-hourly AZT during labour and TDF/FTC post-delivery. There were no women on a PI-based HAART (triple therapy). They were all on efavirenz (EFV) or NVP-based ART as per the
2010 National South African PMTCT guidelines, which were applied by all the surrounding clinics and at the DGMH during the study period. The CD4 cell count was done by the National Health Laboratory System (NHLS) laboratory using a Beckman Coulter (Fullerton, CA) Epics XL MCL cytometer and Beckman Coulter TQ PREP.

\section{Data analysis}

Data were captured by Microsoft Excel ${ }^{\oplus}$ (Microsoft Corp, Redmond, WA, USA) and exported to SPSS ${ }^{\circledR}$ version 20 software (IBM Corp, Armonk, NY, USA) for statistical analysis. The data obtained were analysed using Student's t-test, analysis of variance (ANOVA) as appropriate and the chi-square or Fisher's exact test. Post hoc analysis was performed by using Bonferroni multiple-comparison tests. Statistical significance was considered at $p<0.05$.

\section{Ethical considerations}

Ethics approval was obtained from the School Research Committee and from Sefako Makgatho Health Science Research Ethics Committee. All participants were asked to sign informed consent before participating in the study. Confidentiality and anonymity of the data was ensured by group data analysis without any personal identifiers. The study also obtained full permission from the Head of the Department of Obstetrics and Gynaecology at DGMH.

\section{Results}

A total of 505 of mother and infant pairs were screened during the period of the study. Some $2 \%(9 / 505)$ of mothers and their infants were excluded from the study because of a $22 \%(2 / 9)$ chronic medical condition and $78 \%(7 / 9)$ had twin infants. Of the 496 remaining mothers, 41\% (208/496) tested HIV-negative, while $59 \%(288 / 496)$ were HIV-positive. Of the HIV-positive mothers, $72 \%$ (206/288) were on ART, while $18 \%$ (82/288) were not on ART. The ages of the mothers ranged from 15 to 47 years with a mean of $27.6 \pm 6.5$ years. The mean GA was $35.9 \pm 3.6$ weeks (range 24 to 42 weeks). The infants' birthweights ranged from $550 \mathrm{~g}$ to $4900 \mathrm{~g}$, with a mean birth weight of $2500 \mathrm{~g} \pm 900 \mathrm{~g}$.

Table 1 shows the relationship between maternal HIV status and mother's age, mode of delivery, birthweight and GA. The mean age of HIV-positive mothers on ART or ARV prophylaxis was

Table 1: General characteristics of enrolled mothers and their infants

\begin{tabular}{|c|c|c|c|c|}
\hline Factor & HIV-negative $n=208, n(\%)$ & $\begin{array}{l}\text { HIV-positive on ART/ARV prophylaxis } \\
\qquad n=206, n(\%)\end{array}$ & HIV-positive not on ART $n=82, n(\%)$ & $p$-value \\
\hline Mother's age (years) & $26.34 \pm 6.93$ & $28.63 \pm 6.34$ & $27.76 \pm 6.10$ & $<0.0001$ \\
\hline Delivery by $\mathrm{C} / \mathrm{S}$ & $83(40)$ & $74(36)$ & $7(9)$ & $<0.0001$ \\
\hline \multicolumn{5}{|l|}{ Birthweight } \\
\hline$<1000$ grams & $8(4)$ & $8(4)$ & $10(12)$ & $0.0190^{*}$ \\
\hline 1 000-1 499 grams & $34(16)$ & $21(10)$ & $13(16)$ & 0.1540 \\
\hline $1500-2499$ grams & $37(18)$ & $52(25)$ & $13(16)$ & 0.0930 \\
\hline$\geq 2500$ grams & $129(62)$ & $125(61)$ & $46(56)$ & 0.6330 \\
\hline \multicolumn{5}{|l|}{ Gestational age } \\
\hline$<28$ weeks & $3(1)$ & $2(1)$ & $6(7)$ & 0.0030 \\
\hline 28-29 weeks & $9(4)$ & $13(6)$ & $4(5)$ & 0.6550 \\
\hline 30-36 weeks & $47(23)$ & $50(24)$ & $22(27)$ & 0.7480 \\
\hline$\geq 37$ weeks & $149(72)$ & $138(67)$ & $49(60)$ & 0.1430 \\
\hline
\end{tabular}

Notes: Post hoc analysis: HIV -ve vs. HIV +ve on ART, $p<0.05$; HIV -ve vs. HIV +ve not on ART, $p>0.05 ;$ HIV +ve on ART vs. HIV +ve not on ART, $p>0.05$.

*Fisher's exact test. 
significantly higher than the control group $(p<0.05)$. HIV-negative mothers significantly delivered by CS compared with the other groups $(p>0.05)$. The risk of birthweight $<1000 \mathrm{~g}$ was higher (12\%) amongst HIV-positive mothers not on ART or ARV prophylaxis compared with HIV-positive mothers on ART or ARV prophylaxis $(4 \%)$ and $(4 \%)$ the control group $(p<0.05)$. However, there was no significant difference in infants weighing $\geq 1000 \mathrm{~g}$ among the three groups $(p>0.05)$. A significantly greater proportion (7\%) of mothers not on ART or ARV prophylaxis delivered babies at $\mathrm{GA}<28$ weeks, compared with mothers on ART or ARV prophylaxis (1\%) and (1\%) the control group $(p<0.05)$.

As seen in Table 2, there was no significant relationship between mode of delivery and mother's age and the gestational age of the infant $(p>0.05)$. Infants delivered by CS had significantly higher mean birthweight than those delivered vaginally $(2.68 \pm 0.89$ vs $2.47 \pm 0.91, p<0.05)$. Few $(n=86)$ of the HIVpositive mothers had a CD4 count cell recorded due to the NHLS change of system to Lab Track, which made it impossible to make a meaningful comparison.

\section{Discussion}

From our study, $72 \%$ of the HIV-positive pregnant mothers were on ART, which was comparable to the rate of $72 \%$ found in Kenya $^{12}$ and $82 \%$ found in Ghana. ${ }^{13}$ The report by UNAIDS showed that, in 2013 , only $68 \%$ of HIV-positive pregnant women in sub-Saharan Africa received ART.${ }^{14}$ Evidently, early initiation of ART or ARV prophylaxis during pregnancy has significant clinical benefits. ${ }^{4,5}$ However, these studies show that the uptake of ART among HIV-positive pregnant women in sub-Saharan Africa remains a challenge and is associated with a shortage of healthcare workers, the poor attitudes of healthcare workers, transport costs and long waiting times..$^{15}$ In the present study, HIV-positive mothers on ART or ARV prophylaxis and HIVnegative mothers had a lower risk of premature birth compared with HIV-positive mothers not on ART or ARV prophylaxis.

Overall, nearly half ( $41 \%)$ of the HIV-positive pregnant women in our study delivered LBW babies, which is slightly higher than the rate of LBW babies (34\%) observed in India. ${ }^{16}$ Interestingly, Xiao and co-workers, in their meta-analysis, found that ARV drug usage did not significantly change the association of maternal HIV exposure to ARV drugs with LBW. ${ }^{11}$ In contrast, one study in South Africa found that HIV-positive mothers not on ART or ARV prophylaxis delivered significantly LBW infants compared with the HIV-positive mothers on ART or ARV prophylaxis. ${ }^{17}$ It is worth noting that, in our study, HIV-positive women not on ART or ARV prophylaxis significantly delivered babies with birthweight $<1$ $000 \mathrm{~g}$. The findings in our study could be due to the fact that the

Table 2: Maternal and infant profile by mode of delivery and CD4 count

\begin{tabular}{|l|c|c|c|}
\multirow{2}{*}{ Factor } & \multicolumn{2}{|c|}{ Mode of delivery } & \multirow{2}{*}{ p-values } \\
\cline { 2 - 3 } & $\begin{array}{c}\text { Caesarean } \\
\text { section }\end{array}$ & Vaginal births & \\
\hline$n$ & 174 & 322 & 0.2501 \\
\hline $\begin{array}{l}\text { Mother's age: mean } \\
\text { (SD) [median] }\end{array}$ & $28.2(6.3)[28]$ & $27.5(6.6)[27]$ & 0.6996 \\
\hline $\begin{array}{l}\text { Gestational age: } \\
\text { mean (SD) [median] }\end{array}$ & $36.1(3.2)[36]$ & $35.9(3.7)[35]$ & \\
\hline $\begin{array}{l}\text { Birth weight: mean } \\
\text { (SD) [median] }\end{array}$ & $2.7(0.9)[3]$ & $2.5(0.9)[2]$ & 0.0138 \\
\hline
\end{tabular}

HIV-positive mothers not on ART might have had a lower CD4 cell count and higher HIV RNA viral load, which might have contributed to extremely LBW $(<1000 \mathrm{~g})$ babies. $^{18}$

In our study, 35\% of the HIV-positive pregnant women delivered preterm babies, which is higher than the $11 \%$ reported in Nigeria ${ }^{19,20}$ and $25 \%$ in India. ${ }^{16}$ The reason for the high premature birth rate in our study is unclear, but it could be related to young age, low education levels, no or low pregnancy weight gain and HIV disease stage 2 or more. ${ }^{21}$ Several studies have shown an association between prematurity, low birthweight and CD4 cell count. ${ }^{17,18}$ In our study, few $(n=86)$ HIV-positive pregnant women had their CD4 cell count documented, which made it impossible to make a meaningful comparison.

With regard to the association between CS and maternal HIV status, our findings show a significantly greater proportion of HIV-negative pregnant women delivered by CS compared with HIV-positive pregnant women on ART or those not on ART. ${ }^{22}$ The obstetric reasons for lower CS rate amongst HIV-positive pregnant women in our study were not documented. However, the 2010 South African National Guidelines on HIV Treatment did not recommend an elective Caesarean for the prevention of mother-to-child transmission (PMTCT). ${ }^{23}$ We found no significant difference in the mean age of HIV-positive mothers on ART or ARV prophylaxis and those not on treatment, which is in agreement with previous studies.

\section{Study limitations}

The study did not assess other risk factors associated with preterm delivery, such as socio-economic factors, mother's height, pregnancy-induced hypertension and sexually transmitted disease. The CD4 cell count and the viral load data were missing for most of the HIV-positive women. Few of the HIV-positive mothers had their CD4 count documented, as the NHLS changed its previous electronic results program (DISALAB) to a better computerised program, Hospital Statistics. Because of this changeover, most of the results were missing. We included some of the women on ARV prophylaxis and their babies as exposed to ARV drugs. Lastly, ART drugs were not documented in the admission maternity files, even for those on ARV prophylaxis.

\section{Conclusion}

The findings of this study show that HIV-positive pregnant women not on ART or ARV prophylaxis were at a high risk of delivering babies at $\mathrm{GA}<28$ weeks with birthweight $<1000 \mathrm{~g}$. The first PROMISE study, conducted in 2013, showed a significantly lower early rate of mother-to-child HIV transmission amongst women on lifelong ART/triple therapy (EFV and NVP based) compared with those receiving ARV prophylaxis. ${ }^{24}$ Therefore, since 2013, the World Health Organization (WHO) PMTCT guidelines do not recommend ARV prophylaxis for PMTCT, as was the case in the 2010 WHO PMTCT guidelines. There is a need to encourage early and regular attendance at antenatal care sessions so that HIV-positive pregnant women can be identified and be given access to early treatment and support during pregnancy.

Disclosure statement - No potential conflict of interest was reported by the authors.

Acknowledgement - The authors would like to thank the women and infants who participated in this study. The authors also thank the nurses and doctors and the laboratory technicians at DGMH. 


\section{References}

1. UNAIDS. Global HIV Statistics. Fact sheet November 2016. Available from: at http://www.unaids.org/en/resources/fact-sheet. Accessed 25 May 2017.

2. Department of health. (2015). The national HIV and syphilis prevalence survey South Africa 2013. Available from: https://www. health-e.org.za/wp-content/uploads/2016/03/Dept-Health-HIVHigh-Res-7102015.pdf. Accessed 22 September 2011.

3. Townsend CL, Byrne L, Cortina-Borja M, et al. Earlier initiation of ART and further decline in mother-to-child HIV transmission rates, 20002011. AIDS 2014;28:1049-57. doi:10.1097/QAD.0000000000000212

4. Temprano ANRS. 12136 study group. A trial of early antiretrovirals and isoniazid preventive therapy in Africa. N Engl J Med 2015;373:808-22.

5. INSIGHT START Study Group. Initiation of antiretroviral therapy in early asymptomatic HIV infection. N Engl J Med 2015;373:795-807.

6. Fowler MG, Qin M, Fiscus SA, et al. Benefits and risks of antiretroviral therapy for perinatal HIV prevention. N Engl J Med 2016 Nov 3;375(18):1726-37. https://doi.org/10.1056/NEJMoa1511691

7. Gagnon LH, MacGillivray J, Urquia ML, et al. Antiretroviral therapy during pregnancy and risk of preterm birth. Eur J Obstet Gynecol Reprod Biol. 2016 Jun;201:51-5. doi: 10.1016/j.ejogrb.2016.03.028.

8. Salihu HM, Mogos MF, August EM, et al. HIV infection and its impact on fetal outcomes among women of advanced maternal age: a propensity score weighted matching approach. AIDS Res Hum Retroviruses. 2013 Mar;29(3):581-7. doi:10.1089/AID.2012.0242.

9. Mesfin $Y M$, Kibret KT, Taye A. Is protease inhibitors based antiretroviral therapy during pregnancy associated with an increased risk of preterm birth? Systematic review and a meta-analysis. Reproductive Health. 2016 Apr5;13:30. doi:10.1186/s12978-016-0149-5.

10. Moodley T, Moodley D, Sebitloane $M$, et al. Improved pregnancy outcomes with increasing antiretroviral coverage in South Africa. BMC Pregnancy Childbirth. 2016 Feb 11;16:35. doi:10.1186/s12884016-0821-3.

11. Xiao PL, Zhou YB, Chen Y, et al. Association between maternal HIV infection and low birth weight and prematurity: a meta-analysis of cohort studies. BMC Pregnancy Childbirth. 2015 Oct 8;15:246. doi:10.1186/s12884-015-0684-z.

12. Sirengo M, Muthoni L, Kellogg TA, Kim AA, et al. Mother-to-child transmission of HIV in Kenya: results from a nationally representative study. JAIDS Journal of Acquired Immune Deficiency Syndromes 2014 May 1;66(Suppl 1):S66-74. doi:10.1097/QAI.0000000000000115.

13. Dako-Gyeke P, Dornoo B, Ayisi Addo S, et al. Towards elimination of mother-to-child transmission of HIV in Ghana: an analysis of national programme data. Int J Equity Health. 2016 Jan;13(15):5. doi:10.1186/ s12939-016-0300-5.
14. UNAIDS. The gap report 2014. Retrieved from http://www.unaids.org on [6 June 2016]

15. Colvin CJ, Konopka S, Chalker JC, et al. A systematic review of health system barriers and enablers for antiretroviral therapy (ART) for HIV-infected pregnant and postpartum women. PLoS ONE. 2014;9:e108150. doi:10.1371/journal.pone.0108150.

16. Darak S, Darak T, Kulkarni S, et al. Effect of highly active antiretroviral treatment (HAART) during pregnancy on pregnancy outcomes: experiences from a PMTCT program in Western India. AIDS Patient Care and STDs 2013 Mar;27(3):163-70. doi:10.1089/apc.2012.0401.

17. van der Merwe $K$, Hoffman R, Black V, et al. Birth outcomes in South African women receiving highly active antiretroviral therapy: a retrospective observational study. J Int AIDS Soc. 2011;14:42.10.1186/1758-2652-14-42

18. Yu L, Li WY, Chen RY, et al. Pregnancy outcomes and risk factors for low birth weight and preterm delivery among HIV-infected pregnant women in Guangxi, China. Chin Med J (Engl) 2012 Feb;125(3):403-9.

19. Afe AJ, Adewum N, Emokpa A, et al. Outcome of PMTCT services and factors affecting vertical transmission of HIV infection in Lagos, Nigeria. HIV \& AIDS Review. 2011;10(1):14-8. doi:10.1016/j. hivar.2011.02.001.

20. Ezechi OC, David AN, Gab-Okafor CV, et al. Incidence of and sociobiologic risk factors for spontaneous preterm birth in HIV positive Nigerian women. BMC Pregnancy Childbirth. 2012 Sep 9;12:93 https://doi.org/10.1186/1471-2393-12-93

21. Zack RM, Golan J, Aboud S, et al. Risk Factors for Preterm Birth among HIV-Infected Tanzanian Women: A Prospective Study. Obstet Gynecol Int. 2014;2014:261689. doi:10.1155/2014/261689.

22. Mark S, Murphy KE, Read S, et al. HIV mother-to-child transmission, mode of delivery, and duration of rupture of membranes: experience in the current era. Infect Dis Obstet Gynecol. 2012;2012:267969. doi:10.1155/2012/267969.

23. South African Department of Health. The South African antiretroviral treatment guidelines 2010. Pretoria; 2010. Available from http:// www.sanac.org.za/resources/art-guidelines. Accessed [15 March 2012].

24. World Health Organization. First PROMISE study results confirm WHO recommendations to treat pregnant women and reduce motherto-child-transmission of HIV. 2014 Nov. Available from http://www. who.int/hiv/mediacentre/news/promise-study-result/en/. Accesed 4 March 2017. 